\title{
Особенности ионизации примесей в квазиклассически сильных постоянном и переменном электрических полях в двумерной сверхрешетке на основе графена
}

\author{
(С) П.В. Бадикова ${ }^{1}$, С.Ю. Глазов ${ }^{1,2, \uparrow}$, Г.А. Сыродоев ${ }^{1}$ \\ ${ }^{1}$ Волгоградский государственный социально-педагогический университет, \\ 400005 Волгоград, Россия \\ ${ }^{2}$ Волгоградский государственный медицинский университет, \\ 400131 Волгоград, Россия \\ E-mail: ser-glazov@yandex.ru
}

Поступила в Редакцию 27 декабря 2018 г.

В окончательной редакции 4 февраля 2019 г.

Принята к публикации 6 февраля 2019 г.

Исследован коэффициент поглощения электромагнитной волны в двумерной сверхрешетке на основе графена, в присутствии постоянного электрического поля для случая квазиклассически сильных электрических полей. Выявлена анизотропия коэффициента поглощения при разной ориентации вектора напряженности постоянного и вектора поляризации переменного электрических полей. Показано, что медленный рост коэффициента поглощения при малых частотах обусловлен примесным поглощением, а более быстрый его рост при больших частотах определяется межминизонными переходами. Эти особенности можно использовать при создании детекторов излучения.

Ключевые слова: двумерная графеновая сверхрешетка, ионизация примесей, вероятность ионизации, туннелирование, коэффициент поглощения электромагнитной волны.

DOI: 10.21883/FTP.2019.07.47869.9057

\section{1. Введение}

Электрические и оптические свойства полупроводников в значительной мере определяются наличием в них примесей. Особенно актуальной такая ситуация представляется в сильных электрических полях, которые могут приводить к кардинальной модификации примесных состояний и тем самым динамически изменять концентрацию носителей в зоне проводимости. В данной работе область рассматриваемых частот лежит ниже края фундаментального (однофотонного) поглощения, что позволяет получить количественные данные как о структуре мини-зон, так и о глубине залегания примесей при переходе от туннельного к многофотонному поглощению.

Квазиклассическая теория ионизации атомов под действием внешних электрических полей рассматривается в работах [1-4] с использованием метода „мнимого времени“. В этих работах энергетический спектр носителей заряда в рассматриваемых структурах считался квадратичным. Развитие такого подхода для полупроводниковых материалов с неквадратичным энергетическим спектром выполнено в $[5,6]$.

В последнее время активно изучается графен и структуры на его основе, интерес к которым обусловлен высокой подвижностью носителей заряда, непараболичностью и неаддитивностью энергетического спектра [7]. Причем особое внимание уделяется объектам, в которых энергетический спектр носителей содержит запрещенную зону, графеновым полупроводникам, востребован- ным в современных электронных устройствах. Одной из таких структур является графеновая сверхрешетка (ГСР), которая может быть получена путем создания в образце дополнительного периодического потенциала, с периодом, превышающим период основной решетки. ГСР могут применяться при создании инфракрасных датчиков, фотометрических преобразователей, оптических модуляторов и детекторов. В обзоре [8] представлены различные виды полупроводниковых структур на основе графена, в том числе ГСР с периодически модулированной поверхностью, а также основанные на графеновых лентах. ГСР может быть сформирована под действием пространственно-периодического магнитного поля [9], путем приложения затворного напряжения между подложкой и периодически расположенными на поверхности графена полупроводниковыми контактными площадками [10-13]. В [14] предложена модель сверхрешетки на основе графена, образующаяся за счет периодической модуляции запрещенной зоны. Такая модуляция возможна в графене, осажденном на подложку из периодически чередующихся полосок, например, $\mathrm{SiO}_{2}$ и $\mathrm{SiC}$. Работа [14] положила основу для теоретического изучения различного рода процессов и явлений в такой структуре [15-18].

В последнее время внимание исследователей сосредоточивается на изучении двумерных (2D) ГСР. В [19] сообщается о новом подходе к изготовлению ГСР, в котором сверхрешеточный потенциал модулируется подложкой с периодическими наноотверстиями. В работе [20] исследуется уникальная двумерная сверхрешетка, 
Коэффициенты $f_{i}$ в законе дисперсии (1), $d_{\mathrm{I}}=d_{\mathrm{II}}$

\begin{tabular}{|c|c|c|c|c|c|c|c|}
\hline \multirow{3}{*}{$d, 10^{-6} \mathrm{~cm}$} & \multicolumn{7}{|c|}{$i$} \\
\hline & \multicolumn{2}{|c|}{1} & \multicolumn{2}{|c|}{2} & \multirow{2}{*}{$\frac{3}{\varepsilon_{2}}$} & \multicolumn{2}{|c|}{$\left(\varepsilon-\varepsilon_{\mathrm{app}}\right) / \varepsilon_{\mathrm{app}}, \%$} \\
\hline & $\varepsilon_{1}$ & $\varepsilon_{2}$ & $\varepsilon_{1}$ & $\varepsilon_{2}$ & & $\varepsilon_{1}$ & $\varepsilon_{2}$ \\
\hline 1 & 0.43044 & 0.36680 & 0.84919 & 0.87961 & 0.329238 & 10.4 & 2.6 \\
\hline 2 & 0.42171 & 0.4198 & 0.33179 & 0.33421 & 0.090652 & 1.62 & 0.19 \\
\hline 3 & 0.36427 & 0.36420 & 0.15357 & 0.15374 & 0.026709 & 0.24 & 0.011 \\
\hline 4 & 0.30731 & 0.30731 & 0.07521 & 0.07522 & 0.0079692 & 0.03 & $5.7 \cdot 10^{-6}$ \\
\hline 5 & 0.26126 & 0.26126 & 0.038169 & 0.038170 & 0.0024297 & 0.004 & $2.7 \cdot 10^{-7}$ \\
\hline
\end{tabular}

получаемая в результате осаждения графена на подложку из металлических наношаров. Развитие идеи получения одномерной ГСР на двумерный случай выполнено в работах [21,22], в которых исследуется влияние внешних электрических полей на транспортные свойства 2D ГСР.

Присутствие примесей и дефектов может сильно повлиять на электрические свойства графеновых структур $([23,24]$ и ссылки в них), вызвать существенное изменение его энергетического спектра, в частности, привести к появлению запрещенной зоны в энергетическом спектре. Помимо регулярных примесей, вызывают интерес и исследования случайных редких примесных центров. Так, в работе [25] было изучено влияние одиночных примесей на разогрев графена, найдена скорость передачи энергии от электрона к решетке, рассчитано изменение температуры вокруг такой примеси.

Случайные дефекты, редкие примеси могут приводить к появлению в запрещенной зоне полупроводника примесных уровней, с которых электроны имеют возможность перейти в зону проводимости за счет теплового движения или под воздействием внешних электрических полей.

Вероятность ионизации примеси внешними постоянным и переменным электрическими полями в щелевом графене исследовалась в работах [26,27] методом мнимого времени. В сравнении с такой структурой ГСР имеет схожий энергетический спектр, но более узкие разрешенные мини-зоны, что сильно влияет на кинетические свойства образца.

В данной работе изучается одновременное влияние постоянного и переменного квазиклассически сильных электрических полей на ионизацию примесей в двумерной сверхрешетке на основе графена.

\section{2. Энергетический спектр электронов В 2D графеновой сверхрешетке}

Теоретическое изучение электронных свойств исследуемой структуры облегчается, если в спектре есть запрещенная зона, поскольку в этом случае можно ограничиться однозонным приближением.

Закон дисперсии носителей заряда в 2D ГСР на полосчатой подложке (рис. 1) в одноминизонном при- ближении можно описать следующим выражением [21]:

$$
\varepsilon(\mathbf{p})= \pm \sqrt{\begin{array}{r}
\Delta_{1}^{2}+\Delta_{2}^{2}\left(1-\cos \left(p_{x} d_{1} / \hbar\right)\right)+ \\
+\Delta_{3}^{2}\left(1-\cos \left(p_{y} d_{2} / \hbar\right)\right)
\end{array}}
$$

где $p_{x}, p_{y}-$ компоненты квазиимпульса электрона, $d_{i}=d_{\mathrm{I}}+d_{\mathrm{II}}-$ период ГСР, $d_{\mathrm{I}}$ и $d_{\mathrm{II}}-$ ширины полосок бесщелевого и щелевого графена. Разные знаки относятся к мини-зоне проводимости и валентной мини-зоне. Спектр (1) периодичен по $p_{x}$ и сохраняет неаддитивность, присущую спектру графена.

При малых $p_{y}$ выражение (1) переходит в хорошо известное дисперсионное соотношение для одномерной ГСР [16].

Для аппроксимации зависимости энергетического спектра электронов первой мини-зоны проводимости от компоненты квазиимпульса $p_{\|}$вдоль оси ГСР можно предложить два закона, полученных в приближении сильной связи: первый - в модели, учитывающей влияние только „ближайших соседей“, второй - при выходе за рамки этого приближения:

$$
\varepsilon_{j}\left(p_{\|}, 0\right)=\Delta \sqrt{f_{1}^{2}+f_{2}^{2}\left(1-\cos \left(p_{\|} d / \hbar\right)\right)},
$$

$\varepsilon_{2}\left(p_{\|}, 0\right)=\Delta \sqrt{f_{1}^{2}+f_{2}^{2}\left(1-\cos \left(p_{\|} d / \hbar\right)\right)+f_{3}^{2} \cos \left(2 p_{\|} d / \hbar\right)}$,

где $\Delta-$ полуширина запрещенной зоны щелевой модификации графена, коэффициенты $f_{i}$ подбираются чис-

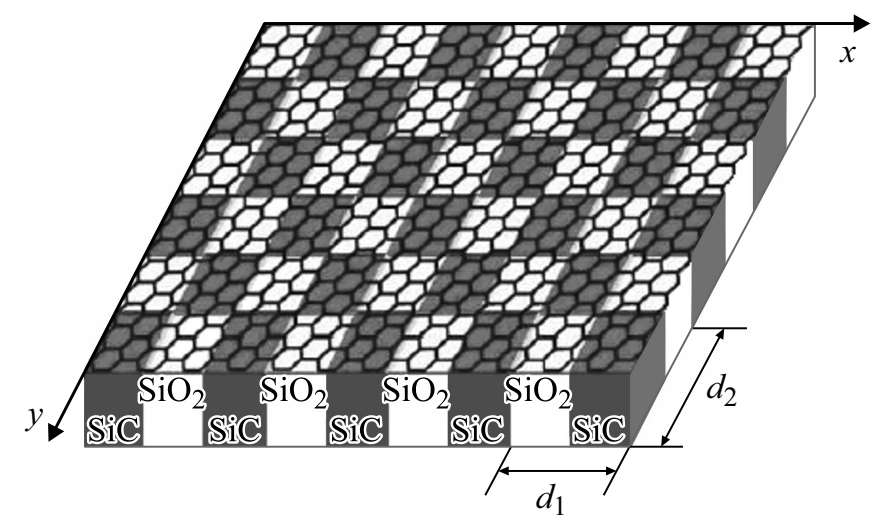

Pис. 1. Двумерная графеновая сверхрешетка. 
ленно на основе непосредственного решения дисперсионного соотношения из [14]. Спектр (2) использовался для описания носителей в ГСР [16,17], но известен еще раньше в теории узкозонных полупроводников и квантовых полупроводниковых сверхрешеток [5].

В таблице приведены значения коэффициентов $f_{i}$ для равной ширины полосок бесщелевой и щелевой модификации графена и максимальная ошибка аппроксимации спектров. Таким образом, можно выбрать вид аппроксимации спектра с нужной точностью. Например, при $d<2 \cdot 10^{-6}$ см спектр (2) уже плохо описывает „истинный“ закон дисперсии электронов первой минизоны и можно воспользоваться более сложной зависимостью (3), содержащей вторую гармонику. При увеличении периода ГСР точность аппроксимации увеличивается. При $d>5 \cdot 10^{-6}$ см $f_{2} \ll f_{1}$ и спектр (2) переходит в аддитивный спектр, хорошо известный в теории полупроводниковых СР [28].

Используя принцип симметрии и известный закон дисперсии носителей заряда для одномерной ГСР, определяем значения параметров в (1) через параметры одномерной ГСР:

$$
\Delta_{1}=\Delta f_{1}\left(d_{1}, d_{2}\right), \quad \Delta_{2}=\Delta f_{2}\left(d_{1}\right), \quad \Delta_{3}=\Delta f_{2}\left(d_{2}\right) .
$$

Для дальнейшего расчета выберем период ГСР $d_{1}=d_{2}=d=3 \cdot 10^{-6}$ см. Для этого случая ширина запрещенной зоны между валентной зоной и зоной проводимости $\varepsilon_{g}=2 \cdot 0.3643 \Delta=0.7286 \Delta$, ширина запрещенной зоны между первой и второй зонами проводимости $\varepsilon_{g 12}=0.6045 \Delta$, ширина первой мини-зоны проводимости $\varepsilon_{e}=0.0598 \Delta$.

\section{3. Вероятность ионизации в случае произвольной ориентации полей}

В сильных полях процесс ионизации примеси представляет собой прохождение через потенциальный барьер и носит квазиклассический характер [1-3]. При этом вероятность перехода (ионизации) может быть записана с экспоненциальной точностью в виде

$$
W=\exp (-2 \operatorname{Im}(S) / \hbar),
$$

где $S$ - классическое действие, набираемое частицей при подбарьерном движении, определяемое формулой

$$
s=\int_{0}^{t_{0}}[\varepsilon(p(t))-V] d t,
$$

где $p(t)$ - импульс электрона, определяемый из классического уравнения движения, $V$ - энергия залегания примеси. Предполагаем, что примеси находятся в графене на определенной глубине $v$ (отсчет ведется от дна зоны проводимости).

Критерием применимости квазиклассического метода является условие

$$
\operatorname{Im}(S) \gg \hbar
$$

Момент начала туннелирования $t_{0}$ находится из условия минимума мнимой части действия

$$
\left.\frac{\partial \operatorname{Im}(S(t))}{\partial t}\right|_{t_{0}}=0
$$

что соответствует условию

$$
\varepsilon\left(t_{0}\right)=V
$$

Рассмотрим наиболее общий случай ориентации полей в плоскости 2D ГСР. Для расчета вероятности перехода электронов с примеси в зону проводимости под влиянием постоянного поля напряженностью $\mathbf{E}_{1}$ и переменного с амплитудой $\mathbf{E}_{0}$ и частотой $\omega$ необходимо решить классическое уравнение движения в проекциях на координатные оси:

$$
\begin{aligned}
& \frac{d p_{x}}{d t}=e E_{1 x}+e E_{0 x} \cos (\omega t), \\
& \frac{d p_{y}}{d t}=e E_{1 y}+e E_{0 y} \cos (\omega t) .
\end{aligned}
$$

Подставляя решения (10) в (9), получим трансцендентное уравнение для нахождения момента начала туннелирования, корень которого чисто мнимый $t_{0}=i \tau_{0}$. После перехода к безразмерным единицам получаем уравнение, которое решается численно:

$$
\operatorname{ch}\left[x\left(\widetilde{\tau}_{0}\right)\right]+\operatorname{ch}\left[y\left(\widetilde{\tau}_{0}\right)\right]=\frac{2 f_{2}^{2}+f_{1}^{2}-\left(f_{1}-\widetilde{v}\right)^{2}}{f_{2}^{2}},
$$

где $\quad x\left(\widetilde{\tau}_{0}\right)=F_{1 x} \widetilde{\tau}_{0}+F_{0 x} \operatorname{sh}\left(w \widetilde{\tau}_{0}\right) / w, \quad y\left(\widetilde{\tau}_{0}\right)=F_{1 y} \widetilde{\tau}_{0}$ $+F_{0 y} \operatorname{sh}\left(w \widetilde{\tau}_{0}\right) / w, \quad \widetilde{v}=v / \Delta, \quad w=\hbar \omega / \Delta, \quad \widetilde{\tau}_{0}=\Delta \tau_{0} / \hbar$, $\mathbf{F}_{1}=\left\{d e E_{1 x} / \Delta, d e E_{1 y} / \Delta\right\}, \mathbf{F}_{0}=\left\{d e E_{0 x} / \Delta, d e E_{0 y} / \Delta\right\}$.

По формуле (6) проведен расчет классического действия, набираемого частицей при подбарьерном движении с момента времени начала туннелирования $t_{0}$ до момента выхода частицы из-под барьера при $t=0$. Подставляя в (6) решения (10), находим мнимую часть действия в виде интеграла

$$
\begin{aligned}
\operatorname{Im} S= & \hbar \int_{0}^{\widetilde{\tau_{0}}}\left\{\sqrt{f_{1}^{2}+f_{2}^{2}(2-\operatorname{ch}[x(t)]-\operatorname{ch}[y(t)])}\right. \\
& \left.+\left(\widetilde{v}-f_{1}\right)\right\} d t,
\end{aligned}
$$

который не выражается через элементарные функции, поэтому берется численно.

На рис. 2 приведена зависимость мнимой части действия от амплитуды переменного электрического поля вдоль оси $X$ при фиксированных значениях напряженности постоянного электрического поля и амплитуды переменного электрического поля вдоль оси $Y$. Выбраны следующие значения параметров: $d=3 \cdot 10^{-6} \mathrm{~cm}$, $d_{\mathrm{I}}=d_{\mathrm{II}}, \Delta=0.13$ эВ, безразмерным напряженности постоянного электрического поля и амплитуде переменного $F_{0,1}=0.01$ соответствуют значения полей 


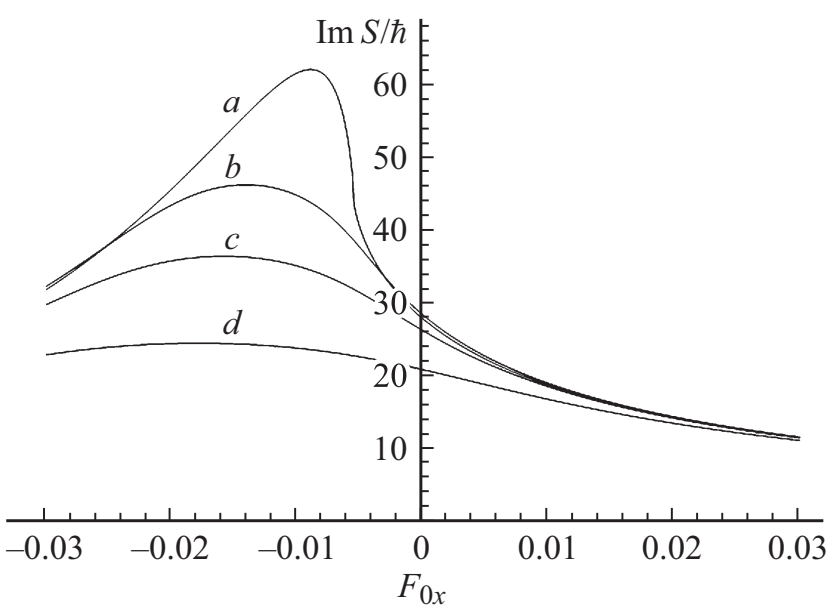

Рис. 2. Зависимость мнимой части действия от амплитуды переменного электрического поля вдоль оси $X$ при: $w=0.01$, $F_{1 x}=0.02, F_{1 y}=0.01 ; a-F_{0 y}=-0.0005 ; b-F_{0 y}=-0.015$; $c-F_{0 y}=-0.02 ; d-F_{0 y}=-0.03, \widetilde{v} \approx 0.8 f_{1}$.

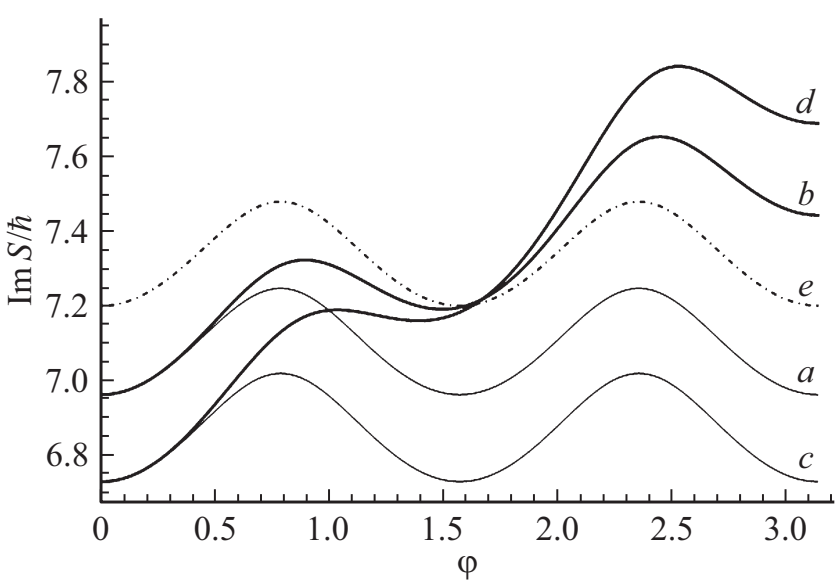

Рис. 3. Зависимость мнимой части действия от угла поворота: сонаправленных постоянного и переменного электрических полей $(a, c)$; переменного электрического поля $(b, d)$, постоянное поле направлено вдоль оси $X$; переменного электрического поля $(e)$, постоянное электрическое поле равно $0 ; w=0.07$, $a, b-F_{0}=0.05, F_{1}=0.005 ; c, d-F_{0}=0.05, \quad F_{1}=0.01$; $e-F_{0}=0.05, F_{1}=0, \widetilde{v} \approx 0.8 f_{1}$.

$E_{0,1} \approx 433 \mathrm{~B} / \mathrm{cm}$, а безразмерной частоте $w=0.01$ соответствует $\omega \approx 2 \cdot 10^{12} \mathrm{c}^{-1}$. Как видно из приведенных графиков, ориентацией полей можно добиться существенного увеличения или уменьшения вероятности ионизации примесей.

Выявлена анизотропия мнимой части действия при разной ориентации вектора напряженности постоянного и вектора поляризации переменного электрических полей. На рис. 3, a, с представлена зависимость мнимой части действия от угла поворота сонаправленных постоянного и переменного электрических полей. Для полей, поляризованных вдоль осей ГСР $(\varphi=0, \pi / 2)$, мнимая часть действия имеет минимальное значение, при $\varphi=\pi / 4$ - максимальное. С увеличением на- пряженности постоянного электрического поля мнимая часть действия уменьшается, что приводит к увеличению вероятности ионизации примеси.

Помимо анизотропии, вызванной геометрией периодического потенциала, создаваемого подложкой, можно задать выделенное направление, зафиксировав положение вектора поляризации одного из электрических полей. Так, на рис. $3, b, d$ приведены графики зависимости мнимой части действия от угла поворота переменного электрического поля, постоянное поле приложено вдоль оси $X$. В отличие от случая, когда постоянное электрическое поле отсутствует $(e)$, наличие постоянного электрического поля приводит к дополнительной анизотропии мнимой части действия, что позволяет добиться существенного увеличения или уменьшения вероятности ионизации примесей, задавая ориентацию переменного электрического поля.

Рассматриваемый в данной работе подход позволяет в зависимости от параметра Келдыша, $\gamma=\omega \tau_{0}$, рассмотреть многофотонное $(\gamma \gg 1)$, туннельное $(\gamma \ll 1)$ поглощения и их совместное проявление $(\gamma \sim 1)$ [1]. С увеличением напряженности постоянного электрического поля значение $\gamma=1$ смещается в область более высоких частот.

Далее рассмотрим частные случаи, когда на 2D ГСР действует только постоянное или только переменное электрические поля.

Когда электрические поля поляризованы вдоль одной из осей 2D ГСР, удается получить аналитический результат в присутствии только постоянного или сильного переменного поля.

Рассмотрим случай, когда на ГСР действует постоянное электрическое поле. Момент начала туннелирования в отличие от общего случая (11) выражается явно

$$
t_{0}=i \frac{2 \operatorname{arcsh}(\xi)}{F_{1}}
$$

где $\xi=\sqrt{\frac{f_{1}^{2}-\left(f_{1}-\widetilde{v}\right)^{2}}{2 f_{2}^{2}}}$.

Классическое действие, набираемое частицей, при такой геометрии задачи имеет вид

$$
S=\frac{2 \hbar f_{1}}{F_{1}}\left\{E(i \xi ; k)+i\left(\widetilde{\mathcal{v}}-f_{1}\right) \operatorname{arcsh}(\xi)\right\} .
$$

После выделения мнимой части действия, используя [29], получаем

$$
\begin{aligned}
\operatorname{Im} S= & \frac{2 \hbar f_{1}}{F_{1}}\{[-E(\operatorname{arctg}(\xi) ; 1-k)+F(\operatorname{arctg}(\xi) ; 1-k) \\
& \left.\left.+\xi \sqrt{\frac{1+k \xi^{2}}{1+\xi^{2}}}\right]+\left(\widetilde{v}-f_{1}\right) \operatorname{arcsh}(\xi)\right\} .
\end{aligned}
$$

Когда на ГСР действует только переменное электрическое поле, „мнимое время“ находится аналитически. 
После введения замены $t_{0}=i \tau_{0}$ и перехода к безразмерным единицам, получаем

$$
\widetilde{\tau}_{0}=\frac{1}{w} \operatorname{arcsh}\left(\frac{2 w}{F_{0}} \operatorname{arcsh}(\xi)\right),
$$

и тогда мнимую часть действия можно представить в виде

$$
\begin{aligned}
\operatorname{Im} S= & \hbar \int_{0}^{\widetilde{\tau}_{0}}\left\{\sqrt{f_{1}^{2}-2 f_{2}^{2} \operatorname{sh}^{2}\left[\left(F_{0} \operatorname{sh}(w t) / w\right) / 2\right]}\right. \\
& \left.+\left(\widetilde{v}-f_{1}\right)\right\} d t .
\end{aligned}
$$

В случае сильного поля формулу (17) с учетом (16) разложим по малому параметру $w / F_{0}$, получим

$$
\begin{aligned}
\operatorname{Im} S & =\hbar\left\{\left(\frac{2 \operatorname{arcsh}(\xi)}{F_{0}}-\frac{4 w^{3} \operatorname{arcsh}(\xi)^{3}}{3 F_{0}^{3}}\right)\left(\widetilde{v}-f_{1}\right)\right. \\
& +\frac{2 f_{1}}{F_{0}}[-E(\operatorname{arctg}(\xi) ; 1-k)+F(\operatorname{arctg}(\xi) ; 1-k) \\
& \left.\left.+\xi \sqrt{\frac{1+k \xi^{2}}{1+\xi^{2}}}\right]-\frac{4 w^{2} f_{1}}{F_{0}^{3}} J(\operatorname{arcsh}(\xi) ; k)\right\}
\end{aligned}
$$

где $F(x ; k)=\int_{0}^{x} \frac{d y}{\sqrt{1-k \sin ^{2}(y)}}-$ эллиптический интеграл I рода, $k=-2 f_{2}^{2} / f_{1}^{2}, E(x ; k)=\int_{0}^{x} \sqrt{1-k \sin ^{2}(y)} d y$ эллиптический интеграл II рода, $J(x ; k)=$ $=\int_{0}^{x} y^{2} \sqrt{1+k \operatorname{sh}^{2}(y)} d y$.

Сравнение (17) с (18) показывает, что расхождение значений, рассчитанных по этим формулам, составляет менее $1 \%$ при $w / F_{0}<0.26$.

Отметим, что получившиеся аналитические законы для мнимой части действия 2D ГСР (15) и (18) аналогичны законам, полученным как для щелевого графена $[26,27]$, так и для СР с неаддитивным спектром.

\section{4. Коэффициент поглощения электромагнитной волны 2D графеновой сверхрешетки при ионизации примесей и межминизонных переходах}

В квазиклассическом приближении межминизонный переход можно представить как составной переход из валентной мини-зоны на примесный уровень (с энергией, точно лежащей на середине запрещенной зоны, в нашем случае равной нулю) и с примесного уровня в мини-зону проводимости. При этом вероятность составного перехода факторизуется, а классическое действие, набираемое частицей при подбарьерном движении, записывается в виде суммы (с учетом симметричности (1))

$$
S=S_{v i}+S_{i c}=2 S_{i c} .
$$

Здесь $S_{v i}$ - действие при переходе „валентная зонапримесный уровень“, $S_{i c}$ - действие при переходе „примесный уровень-зона проводимости“. Выражение для $S_{i c}$ найдено ранее в формуле (12). Таким образом, решение задачи сводится к исследованию ионизации короткодействующего примесного центра в зону, описываемую спектром (1) со знаком „+““. Вероятность ионизации (5) в этом случае определяется выражением

$$
W=\exp \left(-4 \operatorname{Im}\left(S_{i c}\right) / \hbar\right) .
$$

При наличии только постоянного поля удается получить аналитическое выражение для мнимой части действия [5]:

$$
\operatorname{Im} S_{i c}=\frac{2 \hbar f_{1}}{F_{1}} \frac{K(k)-E(k)}{\sqrt{k}},
$$

где $k=1 /\left(1+2 f_{2}^{2} / f_{1}^{2}\right), K(k)$ и $E(k)-$ полные эллиптические интегралы.

Наличие высокочастотного электрического поля приводит к экспоненциальному возрастанию вероятности туннелирования и, следовательно, к экспоненциальному возрастанию коэффициента поглощения света $\alpha$, который может быть записан как отношение энергии, поглощаемой в $1 \mathrm{~cm}^{3}$ за секунду,

$$
W \varepsilon_{g} N /\left(a \tau_{0}\right),
$$

к плотности потока падающей энергии

$$
\sqrt{\chi} \varepsilon_{0} c E^{2} / 2,
$$

где $N$ - число атомов графена на единицу площади, $a-$ толщина графенового слоя [7], $\chi-$ эффективная диэлектрическая проницаемость, $\varepsilon_{0}$ - диэлектрическая постоянная.

В результате коэффициент поглощения электромагнитной волны при ионизации примесей имеет вид

$$
\alpha=\alpha_{0} \frac{\widetilde{v}}{F_{0}^{2} \widetilde{\tau}_{0}} \exp \left(-2 \operatorname{Im} S_{i c} / \hbar\right),
$$

где $\alpha_{0}=\frac{2 e^{2} d^{2} N_{1}}{a \sqrt{x} \varepsilon_{0} c \hbar}, N_{1}-$ число примесных центров на единицу поверхности.

С учетом факторизации вероятности ионизации запишем коэффициент поглощения электромагнитной волны при межминизонных переходах:

$$
\alpha=\alpha_{0} \frac{2 f_{1}}{F_{0}^{2} \widetilde{\tau}_{0}} \frac{N}{N_{1}} \exp \left(-4 \operatorname{Im} S_{i c} / \hbar\right),
$$

Рассмотренные здесь межминизонные переходы электронов в электрическом поле, происходящие за счет туннельного процесса, сопровождаемого поглощением 


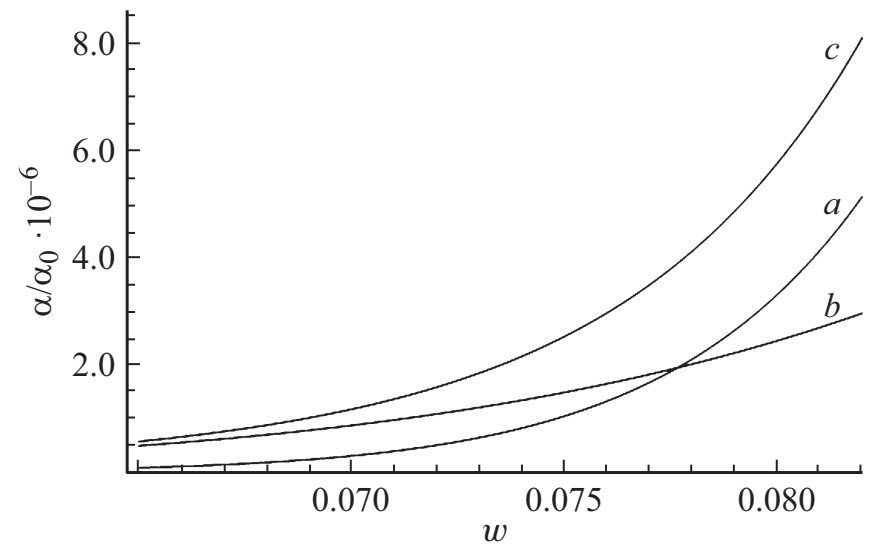

Рис. 4. Зависимость коэффициента поглощения от частоты электромагнитной волны: $a-$ при межминизонных переходах, $N / N_{1}=10^{9} ; b-$ при ионизации примесей $\widetilde{v}=0.85 f_{1} ; c-$ при совместном учете $a$ и $b$.

фотонов с частотой, меньшей края фундаментального поглощения $\hbar \omega<\varepsilon_{g}$, составляют эффект ФранцаКелдыша.

При наличии глубоких примесных центров коэффициент поглощения электромагнитной волны определяется как межминизонными переходами, так и переходами примесь-мини-зона. На рис. 4 показана зависимость коэффициента поглощения от частоты электромагнитной волны при межминизонных переходах $(a)$, при ионизации примеси $(b)$ и при совместном учете влияния этих двух факторов $(c)$. Медленный рост коэффициента поглощения при малых частотах обусловлен ионизацией примесных центров, что характерно и для обычных полупроводниковых гетероструктур. Более быстрый его рост при более высоких частотах определяется межминизонными переходами, поскольку количество электронов, переходящих из валентной мини-зоны в мини-зону проводимости, значительно превышает число электронов, поступающих с примесных центров. При приближении частоты к граничной (однофотонный переход) наблюдается резкое возрастание темпа ионизации как для примесного, так и для межминизонного поглощения. Характер изменения коэффициента поглощения с ростом частоты в эксперименте позволит установить наличие глубоких примесных центров в ГСР.

Рассмотрим поглощение света при межминизонном пробое в 2D ГСР в присутствии постоянного электрического поля. При поглощении света наличие постоянного электрического поля может приводить к увеличению вероятности ионизации. В случае сильных электрических полей электрон, поглощая несколько квантов энергии от переменного электрического поля, переходит в область, где ширина потенциального барьера меньше. В результате достигается экстремальность действия, определяемая условием (8). С ростом постоянного электрического поля происходит увеличение наклона зон и уменьшение ширины области подбарьерного движения, а в области разрешенных зон приводит к квазиклассической локализации электрона.

Когда поля поляризованы вдоль оси $X$, для расчета коэффициента поглощения электромагнитной волны в присутствии постоянного электрического поля необходимо определить ширину барьера в отсутствие переменного поля - $x_{1}$ и найти расстояние пройденное электроном в присутствии обоих полей $x$. Тогда коэффициент поглощения в этом случае может быть оценен соотношением

$$
\alpha=\alpha_{0} \frac{\widetilde{v}}{F_{0}^{2} \widetilde{\tau}_{0}}\left(1-\frac{x}{x_{1}}\right) \exp \left(-2 \operatorname{Im} S_{i c} / \hbar\right),
$$

Фактически речь идет о перенормировке глубины залегания примеси в присутствии постоянного электрического поля.

В случае произвольной ориентации электрических полей расчет перемещения вдоль оси $X$ определяется следующим образом:

$$
\begin{aligned}
x & =\int_{\tau_{0}}^{0} \frac{\partial \varepsilon}{\partial p_{x}} d t \\
& =\frac{d f_{2}^{2}}{2} \int_{0}^{\tilde{\tau}_{0}} \frac{\operatorname{sh}\left[F_{1 x} t+\frac{F_{0 x}}{w} \operatorname{sh}(w t)\right] d t}{\sqrt{f_{1}^{2}+f_{2}^{2}\left(2-\operatorname{ch}\left[F_{1 x} t+\frac{F_{0 x}}{w} \operatorname{sh}(w t)\right]-\right.}},
\end{aligned}
$$

и аналогично рассчитывается перемещение вдоль оси $Y$, что позволяет определить положение точки выхода из-под барьера.

При произвольной ориентации полей удобно повернуть систему координат таким образом, чтобы ось абсцисс совпала с направлением на точку выхода из-под барьера. Это позволяет произвести расчет коэффициента поглощения аналогично (26).

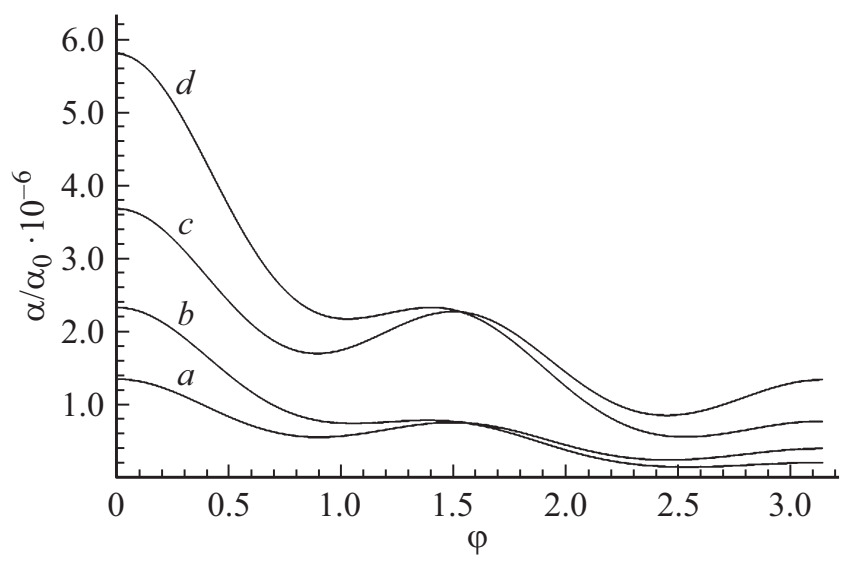

Рис. 5. Зависимость коэффициента поглощения от угла поворота переменного электрического поля: $w=0.06$ : $a-F_{0}=0.05, F_{1}=0.005 ; b-F_{0}=0.05, F_{1}=0.01 ; w=0.07$ : $c-F_{0}=0.05, \quad F_{1}=0.005 ; \quad d-F_{0}=0.05, \quad F_{1}=0.01$, $\widetilde{v} \approx 0.8 f_{1}$. 
Зависимость коэффициента поглощения от угла поворота переменного электрического поля представлена на рис. 5 для фиксированных значений параметров электрических полей. Основной вклад в коэффициент поглощения вносит экспоненциальный член, определяемый мнимой частью действия. Для расчета коэффициента поглощения, приведенного на рис. $5, c$ и $d$, использовалась зависимость мнимой части действия от угла между вектором поляризации переменного электрического поля и направлением постоянного электрического поля (рис. $3, b$ и $d$ ). Параметр Келдыша $\gamma$ для рассмотренных на графиках случаях осциллирует при повороте вектора поляризации переменного электрического поля в диапазоне $[1.7 ; 1.9]$, т.е. при выбранных частотах преобладает комбинированный механизм поглощения.

Выявленные зависимости коэффициента поглощения от угла поворота векторов напряженностей электрических полей могут быть использованы как в устройствах детектирования электромагнитного излучения, так и для уточнения глубины залегания примесных центров.

В данной работе не учитывается рекомбинация носителей заряда, поскольку предполагается импульсный режим облучения образца, для которого длительность импульса больше, чем время туннелирования, а период повторения импульсов гораздо меньше характерного времени рекомбинации.

\section{5. Заключение}

В заключение сформулируем кратко основные результаты и выводы из проделанной работы.

1. Исследована вероятность ионизации примесей в двумерной сверхрешетке на основе графена в присутствии постоянного и переменного внешних электрических полей в квазиклассическом приближении в зависимости от параметров приложенных полей. Показано, что с ростом частоты, амплитуды переменного и напряженности постоянного электрических полей вероятность ионизации примесей в сверхрешетке со щелью увеличивается.

2. Исследован коэффициент поглощения электромагнитной волны в двумерной сверхрешетке на основе графена, в присутствии постоянного электрического поля для случая квазиклассически сильных электрических полей.

3. Выявлена анизотропия коэффициента поглощения при разной ориентации вектора напряженности постоянного и вектора поляризации переменного электрических полей. Показано, что медленный рост коэффициента поглощения при малых частотах обусловлен примесным поглощением, а более быстрый его рост при больших частотах определяется межминизонными переходами.

4. Получено аналитическое выражение для вероятности ионизации примеси и коэффициента поглощения, когда на сверхрешетку действует постоянное или сильное переменное электрическое поле, поляризованное вдоль одной из осей 2D ГСР.

\section{Финансирование работы}

Исследование выполнено при финансовой поддержке РФФИ в рамках научного проекта № 18-42-340005 и Минобрнауки России на выполнение государственных работ в сфере научной деятельности в рамках проектной части государственного задания, код проекта 3.2797.2017/4.6.

\section{Конфликт интересов}

Авторы заявляют, что у них нет конфликта интересов.

\section{Список литературы}

[1] Л.В. Келдыш. ЖЭТФ, 47 (5), 1945 (1964).

[2] В.С. Попов, В.П. Кузнецов, А.М. Переломов. ЖЭТФ, 53, 331 (1967).

[3] А.И. Базь, Я.Б. Зельдович, А.М. Переломов. Рассеяние, реакции и распады в нерелятивистской квантовой механике (М., Наука, 1989).

[4] В.С. Попов, Б.М. Карнаков, В.Д. Мур. ЖЭТФ, 113 (5), 1579 (1998).

[5] С.В. Крючков, Г.А. Сыродоев. Изв. вузов СССР. Радиофизика, 6, 762 (1990).

[6] С.В. Крючков, Г.А. Сыродоев. ФТП, 22 (9), 1695 (1988).

[7] A.H. Castro Neto, F. Guinea, N.M.R. Peres, K.S. Novoselov, A.K. Geim. Rev. Mod. Phys., 81, 109 (2009).

[8] П.Б. Сорокин, Л.А. Чернозатонский. УФН, 183, 113 (2013).

[9] M. Ramezani Masir, P. Vasilopoulos, A. Matulis, F.M. Peeters. Phys. Rev. B, 77, 235443 (2008).

[10] M. Barbier, F.M. Peeters, P. Vasilopoulos, J.M. Pereira,jr. Phys. Rev. B, 77, 115446 (2008).

[11] M. Barbier, P. Vasilopoulos, F.M. Peeters. Phil. Trans. Royal Soc. A, 368, 5499 (2010).

[12] J.M. Pereira,jr, F.M. Peeters, A. Chaves, G.A. Farias. Semicond. Sci. Technol., 25, 033002 (2010).

[13] Li-Gang Wang, Xi Chen. J. Appl. Phys., 109, 033710 (2011).

[14] П.В. Ратников, А.П. Силин. Письма ЖЭТФ, 100 (5), 349 (2014).

[15] S.V. Kryuchkov, E.I. Kukhar'. Physica E, 46, 25 (2012).

[16] Д.В. Завьялов, В.И. Конченков, С.В. Крючков. ФТП, 46 (1), 113 (2012).

[17] С.Ю. Глазов, Н.Е. Мещерякова. Наносистемы: физика, химия, математика, 3 (1), 64 (2012).

[18] С.В. Крючков, Е.И. Кухарь. ФТП, 50 (2), 218 (2016).

[19] C. Forsythe, X. Zhou, K. Watanabe, A. Pasupathy, P. Moon, M. Koshino, P. Kim, C.R. Dean. Nature Nanotechnology, 13, 566 (2018)

[20] Y. Zhang, Y. Kim, M.J. Gilbert, N. Mason. arXiv:1703.05689 [cond-mat.mes-hall] (2018).

[21] S.V. Kryuchkov, C.A. Popov. J. Nano- and Electronic Phys., 9 (2), 02013 (2017).

[22] S.V. Kryuchkov, C.A. Popov. Proc. of the 2017 IEEE 7th Intern. Conf. on Nanomaterials: Application \& Properties (NAP-2017), pt 3 (2017).

[23] M.I. Katsnelson. Graphene: carbone in two dimensions (Cambridge, Cambridge University Press, 2012).

[24] С.Ю. Давыдов. Теория адсорбиии: метод модельных гамильтонианов (СПб., СПбГЭТУ „ЛЭТИ“, 2013). 
[25] K.S. Tikhonov, I.V. Gornyi, V.Yu. Kachorovskii, A.D. Mirlin. Phys. Rev. B, 97, 085415 (2018).

[26] С.Ю. Глазов, П.В. Бадикова. J. Nano- and Electronic Phys., 2 (10), 02020 (2018).

[27] П.В. Бадикова, С.Ю. Глазов. Изв. РАН. Сер. физ., 82 (11), 1536 (2018).

[28] Ф.Г. Басс, А.А. Булгаков, А.П. Тетервов. Высокочастотные свойства полупроводников со сверхрешетками (М., Наука, 1989).

[29] М. Абрамовиц, И. Стиган. Справочник по специальным бункциям (М., Наука, 1979).

Редактор Г.А. Оганесян

\section{Features of impurity ionization in} quasiclassically strong constant and alternating electric fields in a graphene-based two-dimensional superlattice

P.V. Badikova ${ }^{1}$, S.Yu. Glazov ${ }^{1,2}$, G.A. Syrodoev ${ }^{1}$

${ }^{1}$ Volgograd State Socio-Pedagogical University, 400005 Volgograd, Russia

${ }^{2}$ Volgograd State Medical University, 400131 Volgograd, Russia

Abstract The absorption coefficient of an electromagnetic wave in a two-dimensional graphene-based superlattice in the presence of a constant electric field for the case of quasiclassically strong electric fields is investigated. Anisotropy of the absorption coefficient was found for different orientations of the constant vector voltage and the polarization vector of alternating electric fields. It is shown that a slow increase in the absorption coefficient at low frequencies is due to impurity absorption, and it's more rapid growth at high frequencies is determined by interminibands transitions. These features can be used to create radiation detectors. 